\title{
Apports de la cyclostationnarité à l'analyse des signaux mécaniques
}

\author{
JÉRÔME ANTONI ${ }^{\mathrm{a}}$ \\ Laboratoire Roberval (UMR CNRS 6253), Université de Technologie de Compiègne, 60200 Compiègne, France
}

Reçu le 14 septembre 2009, accepté le 22 février 2010

\begin{abstract}
Résumé - La cyclostationnarité, propriété propre aux processus dont les caractéristiques évoluent cycliquement au cours du temps, offre un cadre particulièrement adapté à l'analyse de nombreux signaux acoustiques et vibratoires. Après avoir rappelé les spécificités de l'approche cyclostationnaire dans le contexte mécanique, nous montrons à travers plusieurs exemples les possibilités qu'elle autorise dans des domaines d'application liés au diagnostic des machines, à l'identification des systèmes mécaniques et à la séparation de sources vibro-acoustiques.
\end{abstract}

Mots clés : Cyclostationnarité / signaux cyclostationnaires / diagnostic des machines / identification des systèmes / séparation de sources

Abstract - A cyclostationarity approach to the analysis of mechanical signals. Cyclostationarity offers a theoretical framework to processes whose statistical properties are periodically varying in time. It is found particularly relevant for analysing acoustical and vibration signals. This paper addresses the peculiarities of such an approach. Several examples illustrate its potential to the diagnostics of machines, the identification of mechanical systems and the separation of vibro-acoustical sources.

Key words: Cyclostationarity / cyclostationary signals / machine diagnostics / system identification / source separation

\section{Introduction}

La cyclostationnarité est une propriété qui caractérise les systèmes dont les propriétés statistiques évoluent périodiquement au cours du temps. Initialement développée dans le cadre des signaux de télécommunications [1], elle décrit particulièrement bien un certain nombre de signaux mécaniques. C'est par exemple le cas des signaux vibratoires et acoustiques générés par les machines tournantes et alternatives. Le formalisme cyclostationnaire présente plusieurs avantages pour l'analyse de tels signaux par rapport aux approches classiques. Tout d'abord il permet d'appréhender avec les mêmes outils un large spectre de comportements, depuis la simple périodicité déterministe (phénomènes de balourd, de désalignement, d'excentricité, d'inversion de forces, d'engrènement, ... ) jusqu'à l'aléatoire non-stationnaire (mouvements de fluide, forces de frottement, grippage, ....). Ensuite il intègre explicitement une dimension temporelle qui permet de suivre l'évolution des non-stationnarités des systèmes étudiés.

\footnotetext{
a Auteur pour correspondance : antoni@utc.fr
}

La cyclostationnarité s'applique donc potentiellement à la caractérisation du comportement vibratoire et acoustique de systèmes mécaniques aussi variés que des moteurs thermiques, des machines outils, des réducteurs à engrenages, des compresseurs, des pompes centrifuges, etc. Elle permet d'apporter de nouvelles solutions à un certain nombre de problèmes récurrents pour le diagnostic et l'identification des systèmes mécaniques, ainsi que pour la séparation de sources concurrentes. L'objectif de cet article est précisément de présenter quelques unes des avancées que la cyclostationnarité a récemment autorisées en mécanique. Les exemples sont issus du domaine de l'acoustique et des vibrations, mais n'y sont pas restreints dans le principe. Par ailleurs, l'accent est mis sur la présentation de cas d'études plutôt que sur la description détaillée des algorithmes, dont le lecteur trouvera par ailleurs les références en bibliographie.

\section{Généralités}

De manière traditionnelle, le traitement des signaux mécaniques fait appel aux outils classiques tels que les 


\section{Nomenclature}

\begin{tabular}{|ll|}
\hline$\alpha$ & Fréquence cyclique $(\mathrm{Hz})$ \\
$f S_{x}^{\alpha}(f)$ & Corrélation spectrale du signal $x(t)\left(\mathrm{U}^{2} \cdot \mathrm{Hz}^{-1}\right)$ \\
$H(f)$ & Fréquence porteuse $(\mathrm{Hz})$ \\
$\mathcal{P}\{\bullet\}$ & Réponse fréquentielle \\
$\mathcal{P}\{x(t)\}$ & Opérateur de moyennage cyclique \\
$\mathcal{P}\left\{|x(t)|^{2}\right\}$ & Composante cyclostationnaire à l'ordre $1(\mathrm{U})$ \\
$P_{x}^{\alpha}$ & Puissance instantanée $\left(\mathrm{U}^{2}\right)$ \\
$P_{x}(f)$ & Puissance cyclique $\left(\mathrm{U}^{2}\right)$ \\
$t$ & Densité spectrale de puissance $\left(\mathrm{U}^{2} \cdot \mathrm{Hz}^{-1}\right)$ \\
$\theta$ & Variable temporelle $(\mathrm{s})$ \\
$W V_{x}(t, f)$ & Variable angulaire $($ degrés) \\
$x \Delta f(t ; f)$ & Spectre de Wigner-Ville $\left(\mathrm{U}^{2} \cdot \mathrm{Hz}^{-1}\right)$ \\
$x^{R}(t)$ & Version filtrée du signal $x(t)$ dans une étroite bande \\
$(\mathrm{U}=$ unités du signal $)$ & de fréquence $[f+\Delta f / 2 ; f-\Delta f / 2]$ \\
\hline
\end{tabular}

indicateurs scalaires (valeur moyenne, valeur rms, kurtosis) et vectoriels (densité spectrale de puissance) qui supposent implicitement que les phénomènes étudiés sont stationnaires. Cependant, la stationnarité est souvent plutôt une hypothèse par défaut qu'une hypothèse de travail réaliste. En effet, de nombreux signaux mécaniques - sinon la majorité - sont intrinsèquement non-stationnaires en raison des phénomènes évolutifs qui les génèrent et il s'avère (à l'image des signaux musicaux) que l'information qu'ils véhiculent se situe justement dans cette nonstationnarité. Même si elle simplifie quelque peu les traitements, la stationnarité est incapable de révéler cette information. Au contraire, la cyclostationnarité permet d'en tirer pleinement parti dans le cas particulier où elle provient de phénomènes cycliques ou répétitifs. Il est alors possible d'adjoindre aux indicateurs classiques une dimension supplémentaire qui traduit l'évolution temporelle des phénomènes en synchronisation avec la cinématique de la machine ou, de manière duale, une « fréquence cyclique » qui traduit les jeux de modulation.

Exemple 1 : signal acoustique d'un moteur Diesel. À titre d'exemple, la figure 1a représente un signal de pression acoustique mesuré à $1 \mathrm{~m}$ d'un moteur Diesel 1,9 L, 4 cylindres, tournant au ralenti $\left(735\right.$ tr.min $\left.{ }^{-1}\right)$. Il s'agit d'un archétype de signal cyclostationnaire, qui présente quatre bouffées d'énergie par cycle moteur liées au bruit rayonné par les combustions successives dans les quatre postes. Supposer ce signal stationnaire, comme cela est implicitement pratiqué en analyse spectrale classique, reviendrait à ignorer les relations de phases qui existent entre les composantes fréquentielles, de sorte que le signal serait supposé statistiquement équivalent à celui obtenu en lui attribuant une phase aléatoire, identiquement et uniformément distribuée entre 0 et $2 \pi$, tel que représenté en figure $1 \mathrm{~b}$. Il est clair à travers cet exemple que l'hypothèse de stationnarité « efface » irrémédiablement l'information contenue dans le signal (instants d'occurrence, amplitude et contenu fréquentiel des transitoires) et qu'une approche prenant explicitement en compte la non-stationnarité cyclique du signal a)

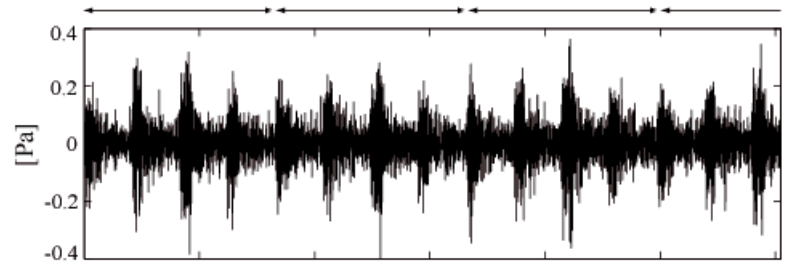

b)

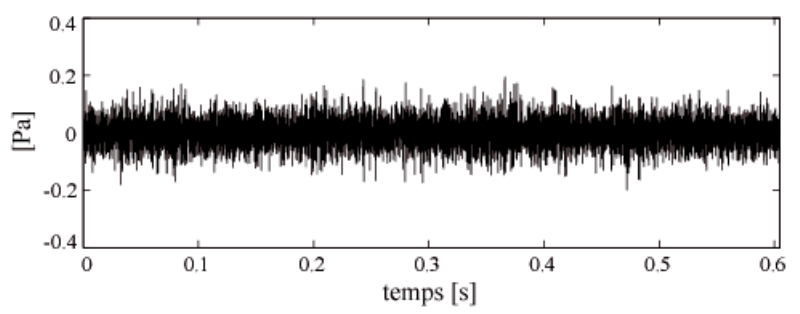

Fig. 1. (a) Pression acoustique mesurée à $1 \mathrm{~m}$ d'un moteur Diesel, 1,9 L, 4 cylindres, représentée sur 3,5 cycles. (b) Signal « stationnarisé » par attribution d'une phase aléatoire identiquement et uniformément distribuée entre 0 et $2 \pi$.

est nécessaire pour « faire parler » les bouffées d'énergie, ici parfaitement visibles, sur la trace temporelle.

À l'issue de ce premier exemple, il est intéressant d'étudier les implications de l'approche cyclostationnaire sur la décomposition de l'énergie (par exemple vibratoire ou acoustique) véhiculée par un signal au cours du temps. Cet exercice permet d'introduire les différents outils que nous utiliserons par la suite.

\subsection{Opérateur de moyennage cyclique}

En premier lieu, il s'agit de définir un opérateur d'extraction des composantes périodiques. L'analyse des signaux stationnaires se fonde sur la notion de moyenne temporelle qui permet par exemple d'extraire l'amplitude moyenne d'un signal (paramètre de position) et sa valeur efficace (paramètre de dispersion). Selon le même principe, l'analyse des signaux cyclostationnaires repose sur la notion de moyenne cyclique. Étant donné A un 

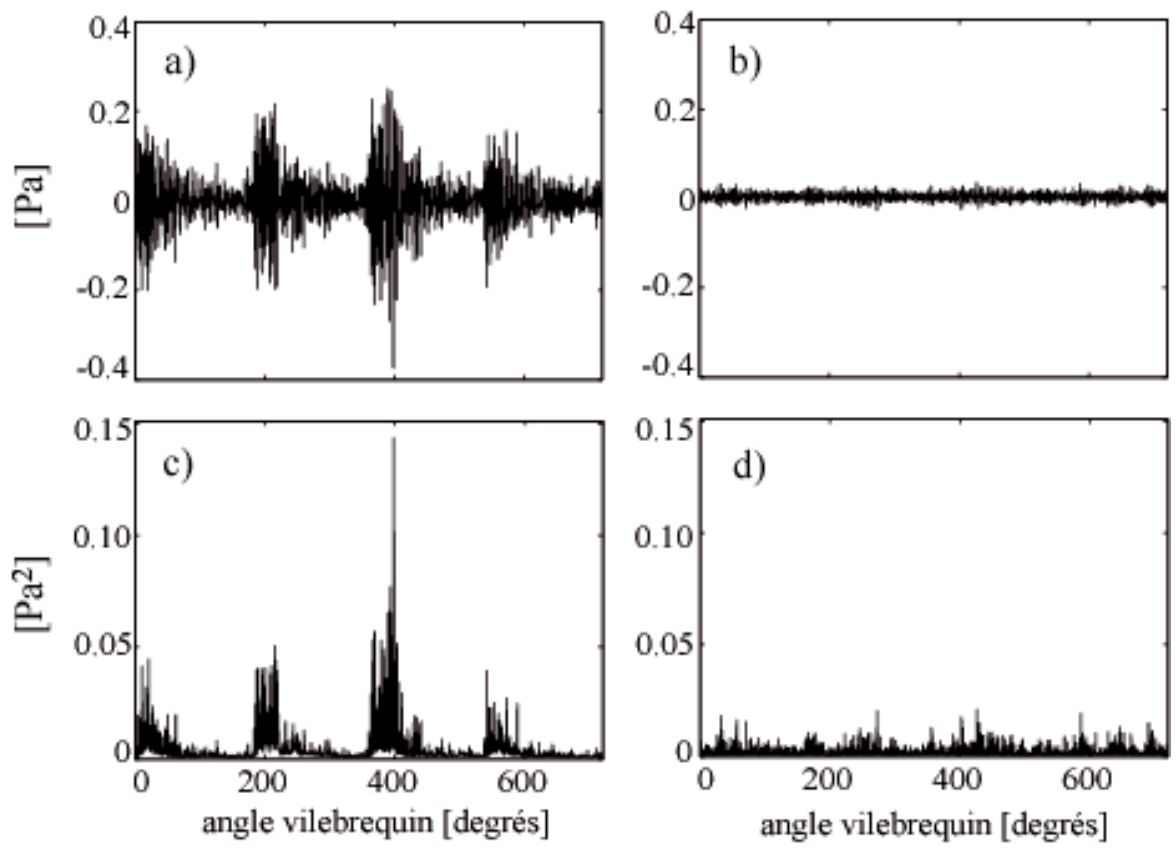

Fig. 2. (a) Moyenne synchrone du signal de pression acoustique de la figure 1a. (b) Moyenne synchrone du signal de pression acoustique de la figure 1b. (c) Puissance cyclique du signal de pression acoustique de la figure 1a. (d) Puissance cyclique du signal de pression acoustique de la figure $1 \mathrm{~b}$.

ensemble de fréquences $\alpha$ qui caractérisent la périodicité d'un mécanisme sous-jacent, l'opérateur de moyennage cyclique $\mathcal{P}$ se définit comme :

$$
\mathcal{P}\{\bullet\}=\sum_{\alpha \in \mathcal{A}} \mathcal{P}_{\alpha}\{\bullet\} \mathrm{e}^{j 2 \pi \alpha t}
$$

où $\mathcal{P}_{\alpha}\{\bullet\}=\lim _{T \rightarrow \infty} \frac{1}{T} \int_{T} \bullet \mathrm{e}^{-j 2 \pi \alpha t} \mathrm{~d} t$ est le coefficient de Fourier à la fréquence $\alpha$. Appliquée directement à un signal $x(t)$, la moyenne cyclique $\mathcal{P}\{x(t)\}$ consiste donc à en extraire l'ensemble de ses composantes périodiques, c'est-à-dire son comportement déterministe. Appliquée à une forme quadratique du signal, elle permet d'extraire les modulations cycliques qui animent sa composante aléatoire, comme illustré ci-dessous. Il est important de noter à ce stade que l'opérateur de moyennage cyclique extrait un signal contrairement à l'opérateur de moyennage temporel classique qui extrait un scalaire.

Exemple 1 (suite). La notion de moyenne cyclique est illustrée ici sur le signal de pression acoustique de l'exemple 1. La figure 2a représente la moyenne $\mathcal{P}\{x(t)\}$, aussi dite moyenne synchrone, calculée sur 200 cycles moteur en prenant $\alpha=1 / 2 / \Omega$ où $\Omega$ est la vitesse de rotation $\mathrm{du}$ moteur. La représentation d'une seule période, par exemple en fonction de l'angle vilebrequin (un cycle $=2$ tours moteur $=\left[0 ; 720^{\circ}\right]$ ), suffit puisque $\mathcal{P}\{x(t)\}$ est périodique par construction. La moyenne cyclique $\mathcal{P}\left\{|x(t)|^{2}\right\}$, dite puissance cyclique, a également été représentée à la figure $2 \mathrm{c}$ pour rendre compte des composantes énergétiques qui se répètent à l'identique d'un cycle à l'autre. Pour comparaison, les figures $2 \mathrm{~b}$ et $2 \mathrm{~d}$ représentent la moyenne synchrone et la puissance cyclique calculées sur le signal stationnarisé de la figure 1b qui, sans surprise, tendent vers des comportements constants (des scalaires) au cours du cycle théoriquement égaux à $\mathcal{P}_{0}\{x\}=\bar{x}$ et $\mathcal{P}_{0}\left\{|x(t)|^{2}\right\}=\overline{x^{2}}$.

\subsection{Les différentes facettes de l'énergie}

Le flux d'énergie véhiculé par un signal $x(t)$ à un instant $t$, c'est-à-dire sa puissance instantanée, s'évalue par la forme quadratique $|x(t)|^{2}$. La moyenne cyclique de la puissance instantanée, dite puissance cyclique, $\mathcal{P}\left\{|x(t)|^{2}\right\}$, rend compte des fluctuations périodiques de l'énergie voir exemple 1. Elle se représente de manière duale par ses coefficients de Fourier $P_{x}^{\alpha}$ qui traduisent l'intensité des composantes périodiques de l'énergie aux fréquences cycliques $\alpha$. Il est intéressant de décomposer ces quantités également en fréquence, au moyen de la corrélation spectrale. Pour cela introduisons $x_{\Delta f}(t ; f)$ la version filtrée du signal $x(t)$ dans une étroite bande de fréquence $[f+\Delta f / 2$; $f-\Delta f / 2]$. La corrélation spectrale se définit alors comme :

$$
C S_{x}^{\alpha}(f)=\lim _{\Delta f \rightarrow 0} \frac{1}{\Delta f} \mathcal{P}_{\alpha}\left\{x_{\Delta f}(t ; f+\alpha / 2) \overline{x_{\Delta f}(t ; f-\alpha / 2)}\right\}
$$

Il s'agit d'une fonction de deux variables fréquentielles : la fréquence porteuse $f$ et la fréquence cyclique $\alpha$. Une valeur non-nulle de la corrélation spectrale traduit l'existence dans le signal d'un paquet d'ondes porté par la fréquence $f$ et dont l'énergie fluctue périodiquement à la cadence $\alpha$. L'interprétation de la corrélation spectrale est donc celle d'une distribution en deux dimensions de paquets d'ondes cyclostationnaires élémentaires qui constituent un signal, tel que schématisé à la figure 3 . 

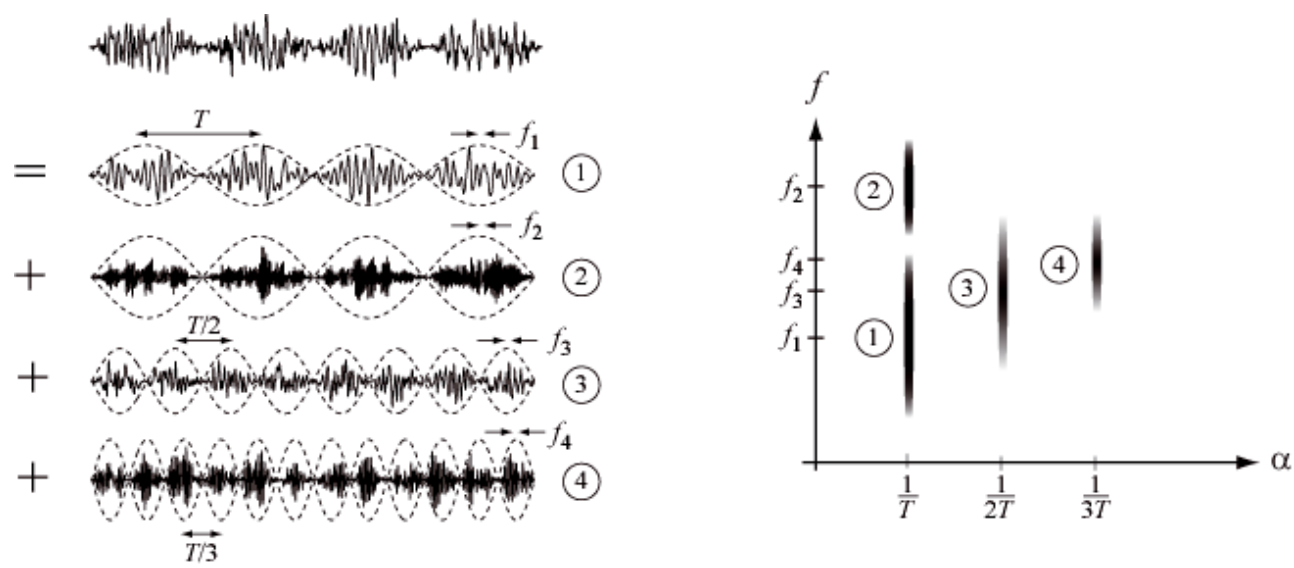

Fig. 3. Interprétation de la corrélation spectrale comme une distribution $2 \mathrm{D}$ des paquets d'ondes de fréquence porteuse $f$ et de fréquence de battement $\alpha$ qui composent un signal cyclostationnaire.

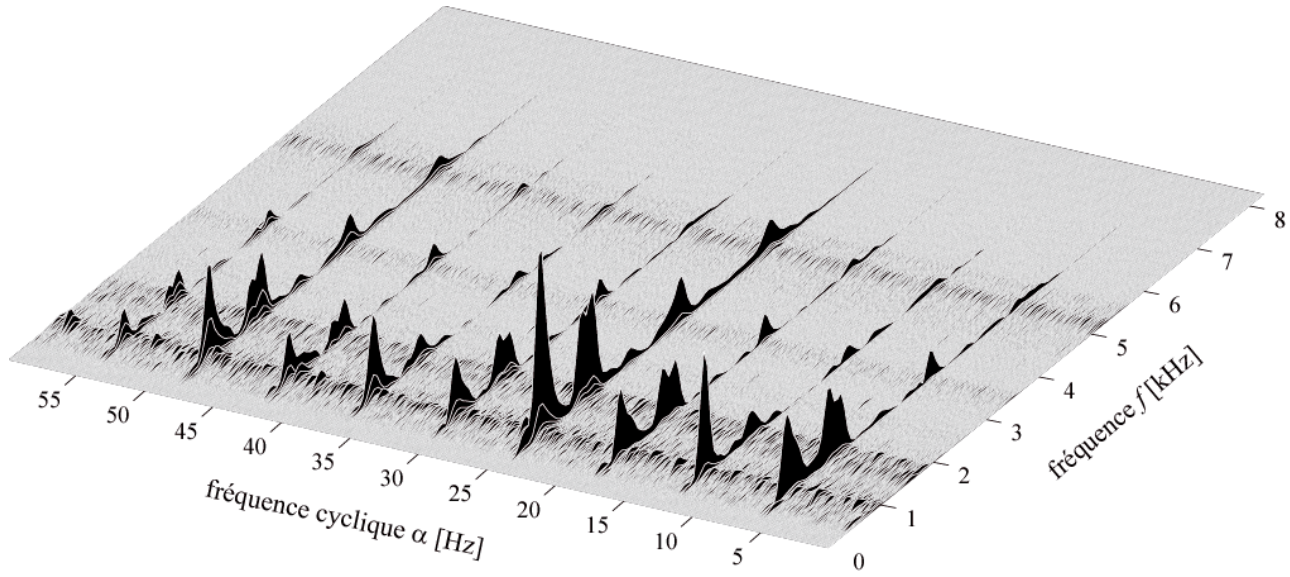

Fig. 4. Corrélation spectrale du signal de la figure 1a $(\Delta f=64 \mathrm{~Hz} ; \Delta \alpha=0,06 \mathrm{~Hz})$.

La corrélation spectrale jouit en outre des propriétés suivantes :

(i) son évaluation en $\alpha=0$ s'identifie à la densité spectrale de puissance - $C S_{x}^{0}(f) \equiv P_{x}(f)$ - telle que classiquement utilisée en analyse des signaux stationnaires (les signaux stationnaires n'ont leur énergie distribuée qu'en la fréquence cyclique $\alpha=0$ ) ;

(ii) son intégration suivant la fréquence $f$ s'identifie aux coefficients cycliques de la puissance instantanée : $\int C S_{x}^{\alpha}(f) \mathrm{d} f=P_{x}^{\alpha}$

(iii) sa sommation en série de Fourier permet de définir une distribution d'énergie temps-fréquence

$$
W V_{x}(t, f)=\sum_{\alpha \in \mathcal{A}} C S_{x}^{\alpha}(f) \mathrm{e}^{j 2 \pi \alpha t}
$$

que nous appellerons par la suite spectre de WignerVille.

Exemple 1 (suite). La corrélation spectrale du signal de pression acoustique de la figure 1a est représentée cidessous à la figure 4 . Elle met très clairement en évidence une fréquence cyclique fondamentale à $\alpha=1 / 2 \Omega=$ $6,1 \mathrm{~Hz}$ et ses multiples harmoniques qui traduisent les modulations engendrées par le cycle moteur, en particulier sur des résonances de structure vers $1 \mathrm{kHz}, 1,8 \mathrm{kHz}$, $3,5 \mathrm{kHz}$ et $5,1 \mathrm{kHz}$. Une forte modulation est également identifiée pour $\alpha=2 \Omega=24,5 \mathrm{~Hz}$ qui correspond à la fréquence d'allumage des postes et dont l'intensité est prédominante, en accord avec l'analyse de la trace temporelle de la figure 1a.

Exemple 2 : analyse cyclostationnaire d'un signal de pompe. Cet exemple a pour but d'illustrer les outils de base de la cyclostationnarité au travers de l'analyse d'un signal vibratoire de pompe centrifuge (45 kW, $\left.2950 \mathrm{tr} \cdot \mathrm{min}^{-1}, 250 \mathrm{~m}^{3} \cdot \mathrm{h}^{-1}\right)$. Un enregistrement de $40 \mathrm{~ms}$ du signal est illustré à la figure 5 a qui révèle un comportement aléatoire sans structure périodique apparente. L'analyse spectrale du signal (non reportée ici) confirme cette observation au travers d'une densité spectrale de puissance « continue » et exempte de raies spectrales. La puissance cyclique $\mathcal{P}\left\{|x(t)|^{2}\right\}$ du signal représentée en trait rouge à la figure $5 \mathrm{a}$ permet par contre de révéler très nettement la présence d'une périodicité cachée dans le signal, ou plus exactement dans son transfert d'énergie. Les coefficients de Fourier correspondants, $P_{x}^{\alpha}$ représentés à la figure $5 \mathrm{~b}$, indiquent un fondamental à $393 \mathrm{~Hz}$ et deux harmoniques à $786 \mathrm{~Hz}$ et $1179 \mathrm{~Hz}$ : il s'agit des fréquences 
(a)
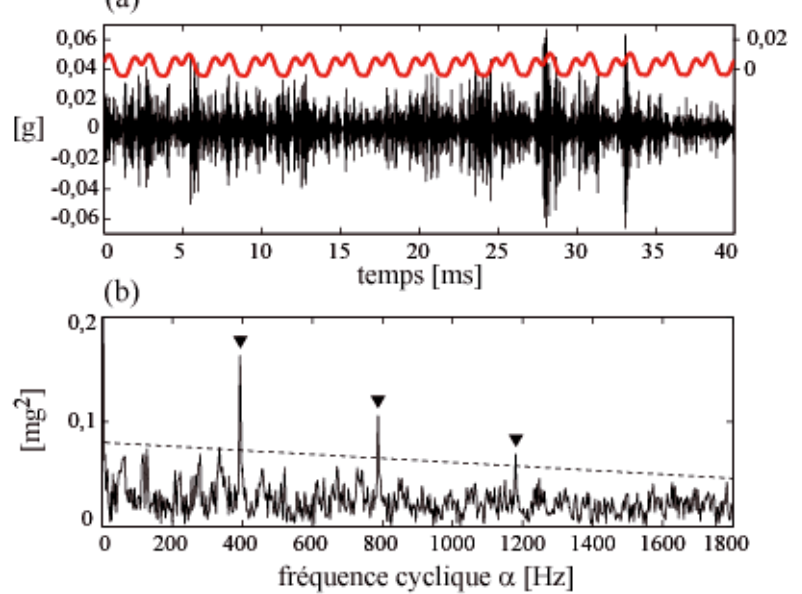

Fig. 5. (a) Signal vibratoire d'une pompe centrifuge et puissance cyclique associée (en rouge). (b) Coefficients de Fourier de la puissance cyclique en fonction de la fréquence cyclique $\alpha$.

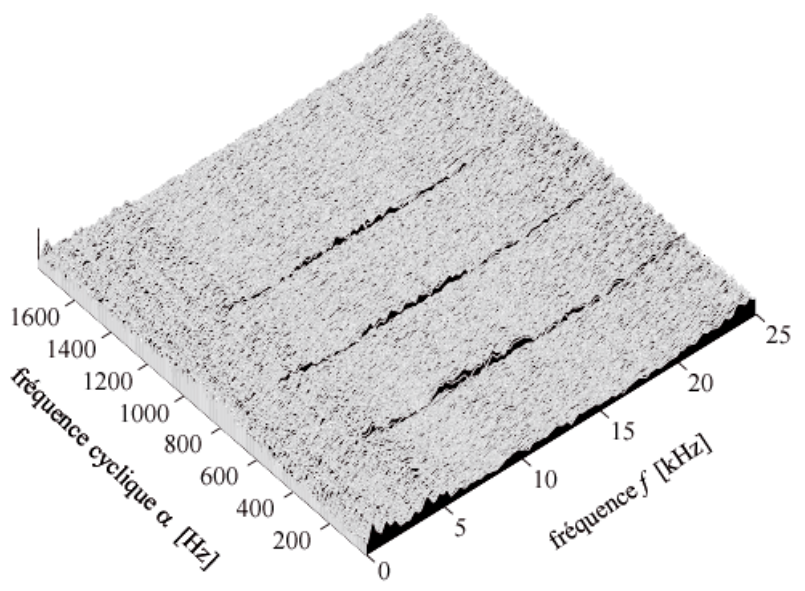

Fig. 6. Corrélation spectrale du signal vibratoire de pompe centrifuge $(\Delta f=62 \mathrm{~Hz} ; \Delta \alpha=2,5 \mathrm{~Hz})$.

de passage des huit aubes de la pompe qui viennent moduler le flux large-bande de fluide dans la pompe. Cette observation se trouve confirmée par la corrélation spectrale à la figure 6 qui montre clairement la nature largebande en $f$ de la porteuse et les fréquences discrètes de la modulation en $\alpha=393 k \mathrm{~Hz}, k=1,2,3$.

\section{Spécificité des signaux vibratoires et acoustiques}

Le traitement cyclostationnaire des signaux vibratoires et acoustiques requiert certaines précautions en raison des spécificités qui les différencient, par exemple, des signaux de télécommunications.

\subsection{Cyclostationnarité d'ordres 1 et 2}

La cyclostationnarité d'ordre 1 est relative à toute composante non-nulle extraite par l'opérateur $\mathcal{P}\{x(t)\}$.
En d'autres termes, $\mathcal{P}\{x(t)\}$ regroupe toutes les contributions périodiques, et donc déterministes, du signal $x(t)$. Il en résulte que le signal résiduel $x^{R}(t)=x(t)-\mathcal{P}\{x(t)\}$ représente la composante purement aléatoire du signal $x(t)$ purifié de l'ordre 1 . La cyclostationnarité d'ordre 2 dite « pure» est alors relative à toute composante nonnulle extraite par l'opérateur $\mathcal{P}\left\{\left|x^{R}(t)\right|^{2}\right\}$ ou, de manière plus générale, par la corrélation spectrale $C S_{x^{R}}^{\alpha}(f)$. Les systèmes mécaniques produisent rarement des signaux purement cyclostationnaires à l'ordre 1 ou 2, mais plutôt une combinaison des deux ordres. Il est avantageux d'analyser ces deux types de cyclostationnarité séparément, car ils sont susceptibles d'être générés par des phénomènes d'origines physiques différentes : ainsi la cyclostationnarité d'ordre 1 est-elle plutôt issue de phénomènes « macroscopiques » de nature déterministe (ondes modales, balourds, excentricités, engrènement) et la cyclostationnarité d'ordre 2 issue de phénomènes « microscopiques » de nature aléatoire (ondes ultrasonores, impacts, frottements, roulement).

Exemple 3 : séparation de la cyclostationnarité d'ordres 1 et $\mathbf{2}$. Les vibrations des engrenages sont généralement dominées par une forte composante cyclostationnaire d'ordre 1. Au contraire, les vibrations des paliers à éléments roulants sont généralement dominées par une composante cyclostationnaire d'ordre 2 [5]. Ces propriétés peuvent être utilisées pour séparer les deux phénomènes dans les systèmes qui font intervenir les deux types de mécanismes [3]. La figure 8 illustre ce principe pour l'analyse du signal vibratoire (soigneusement rééchantillonné en angulaire comme expliqué dans Sect. 3.2) d'un réducteur à engrenages représenté à la figure $7 \mathrm{a}$, dont l'un des paliers à roulements contient un défaut localisé sur sa bague interne - cf. figure 7b. Les chocs transitoires générés par le contact des éléments roulants sur le défaut sont clairement visibles sur la partie cyclostationnaire à l'ordre 2 de la figure $8 \mathrm{c}$.

Exemple 4 : bruit tonal et large bande d'un ventilateur. Le bruit rayonné par un ventilateur possède une structure spectrale très diversifiée en fonction des mécanismes sources qui le génèrent : les forces tournantes qu'appliquent les pales sur le milieu fluide créent un bruit de nature tonal, tandis que les effets de couches limites sur les pales créent un bruit large bande. La décomposition du bruit total rayonné en ses composantes déterministes et aléatoires permet précisément de séparer ces deux types de bruit, comme illustré à la figure 9 dans le cas d'un ventilateur à sept pales tournant à $50 \mathrm{~Hz}$. À noter que dans cet exemple, c'est le bruit large bande qui prédomine globalement et qu'il conviendra de diminuer dans une optique de confort acoustique.

Il est clair que pour atteindre la finesse des résultats présentés dans les exemples 3 et 4 ci-dessus, il est nécessaire que la vitesse de rotation de la machine étudiée soit parfaitement stable. Si cette condition n'est pas vérifiée en pratique, il est possible de la « forcer » à l'aide d'un pré-traitement des signaux décrit dans la section suivante. 
a)

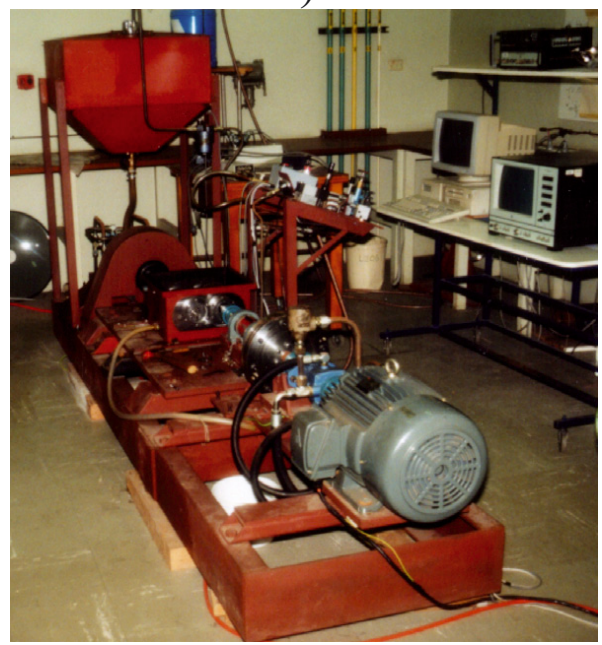

b)

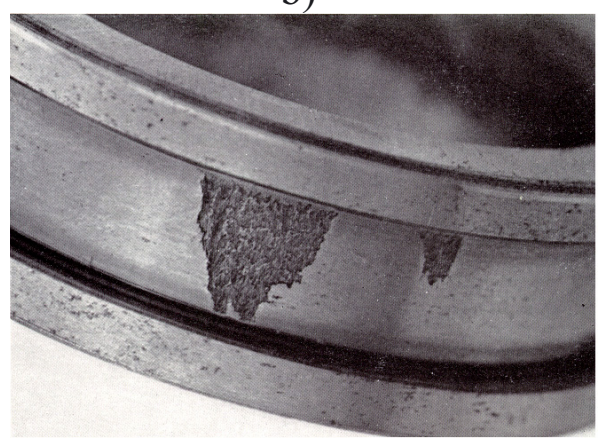

Fig. 7. (a) Réducteur à engrenage avec (b) défaut introduit sur la bague interne d'un des paliers à roulements (University of New South Wales, Sydney). (Reproduit avec l'aimable autorisation du Prof. B. Randall.)

\subsection{Cyclostationnarité temporelle et angulaire}

La cyclostationnarité des signaux mécaniques provient essentiellement des modulations imprimées par la présence de cycles machine et d'organes mécaniques tournants. En ce sens, la cyclostationnarité est à strictement parler relative à une variable d'angle machine au lieu de la variable temporelle. Par ailleurs, on montre que la sensibilité de l'opérateur de moyennage cyclique introduit dans la section 2.1 par rapport à des fluctuations de vitesse de la machine se traduit par un effet de filtrage passe-bas; très précisément, étant donnée la densité de probabilité $\phi(t)$ des fluctuations de vitesse autour de la vitesse nominale et $\Phi(\alpha)$ sa transformée de Fourier en la fréquence $\alpha$, l'équation (1) se réécrit

$$
\mathcal{P}\{\bullet\}=\sum_{\alpha \in \mathcal{A}} \Phi(\alpha) \mathcal{P}_{\alpha}\{\bullet\} \mathrm{e}^{j 2 \pi \alpha t}
$$

où $\Phi(\alpha)$ atténue les composantes $\mathcal{P}_{\alpha}\{\bullet\}$ de façon croissante en fonction de $\alpha$. Par conséquent, afin de conserver intacte la propriété de cyclostationnarité, il peut être avantageux d'échantillonner (directement ou a posteriori)

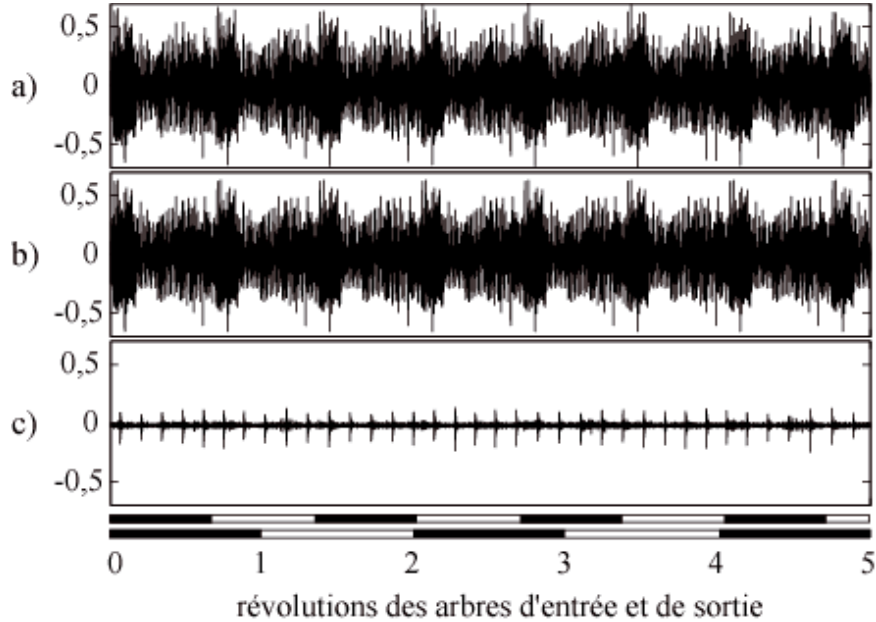

Fig. 8. Décomposition d'un signal vibratoire $x(t)$ de réducteur à engrenages (a) en sa composante déterministe $\mathcal{P}\{x(t)\}$ liée au bruit d'engrènement (b) et en sa composante aléatoire $x^{R}(t)=x(t)-\mathcal{P}\{x(t)\}$ liée au bruit de roulement (c).

a)

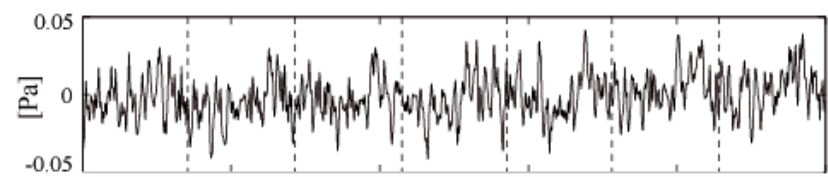

b)

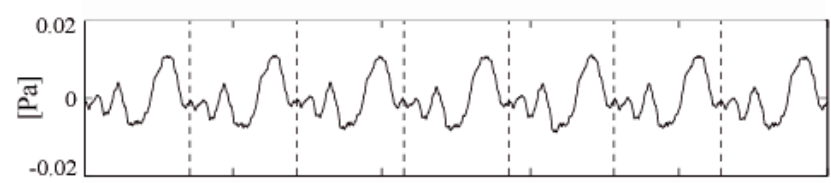

c)

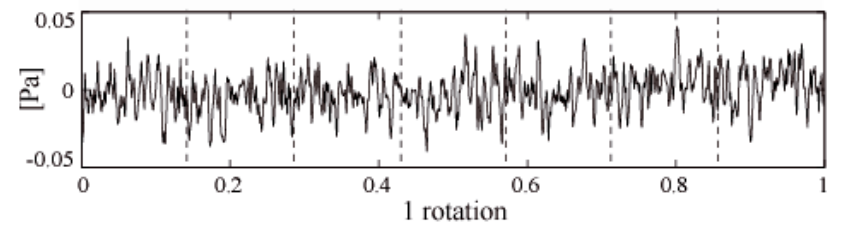

Fig. 9. Décomposition d'un signal acoustique de ventilateur à 7 pales représenté sur une rotation (a) en sa composante déterministe liée au bruit tonal (b) et en sa composante aléatoire liée au bruit large bande (c).

les signaux dans le domaine angulaire à l'aide d'une référence de phase, telle que fournie par exemple par un codeur incrémental. La figure 10 illustre le principe du rééchantillonnage angulaire réalisé a posteriori à l'aide d'une telle référence. Ce prétraitement est tout à fait analogue à celui qui se pratique pour l'analyse d'ordre dans le domaine automobile. Ce pré-conditionnement a été utilisé dans la plupart des exemples présentés dans cet article.

À titre d'exemple, la figure 11 illustre « l'effet passebas $»$ obtenu sur la moyenne synchrone du signal de pression acoustique de l'exemple 4 lorsque réalisée directement dans le domaine temporel, sans rééchantillonnage angulaire préalable. 


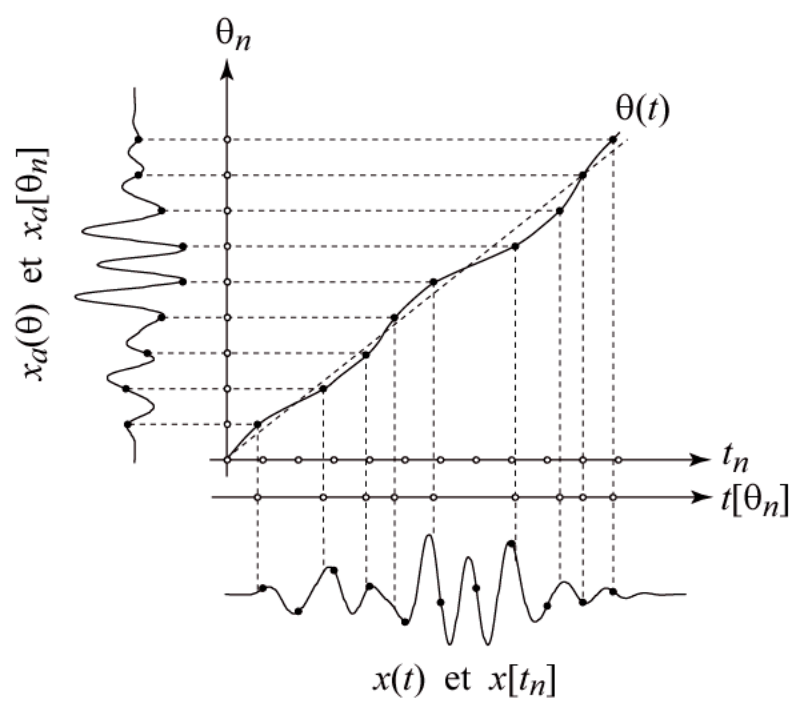

Fig. 10. Principe du rééchantillonnage d'un signal $x$, échantillonné à pas de temps constants $\left(t_{n}=n \Delta t\right)$, en un signal $x_{a}$ à pas d'angle constants $\left(\theta_{n}=n \Delta \theta\right)$, à l'aide de la référence de phase $\theta(t) .(x(\cdot)$ réfère au signal continu et $x[\cdot]$ à la séquence de ses échantillons).

\section{Application au diagnostic des machines}

Le diagnostic des machines, dont l'objectif est d'établir un «état de santé » à partir de symptômes externes, est probablement le domaine d'application mécanique qui, à ce jour, a le plus bénéficié des apports de la cyclostationnarité; [3-6, 10-14] tout d'abord parce que celle-ci est parfaitement adaptée à la détection de défauts qui se manifestent par une libération répétitive de l'énergie vibratoire ou acoustique; ensuite parce qu'elle permet de localiser précisément ces défauts au sein du cycle des machines et que la synchronisation sur des organes tournants distincts permet d'en identifier l'origine même dans des systèmes relativement complexes; enfin parce que la dimension supplémentaire apportée par la cyclostationnarité (axe temporel ou axe dual de la fréquence cyclique) permet une meilleure caractérisation des signatures mécaniques (symptômes) qui peuvent être utilisées dans un système de décision automatique.

Exemple 5 : surveillance d'un compresseur. Cet exemple illustre l'application du spectre de Wigner-Ville - équation (3) - à la surveillance d'un compresseur alternatif à double effet schématisé à la figure 12. Les compresseurs alternatifs se caractérisent par un comportement vibratoire complexe en raison de la multitude des évènements transitoires qui se succèdent dans le cycle machine. Par conséquent leur surveillance reste un exercice difficile, à moins qu'une approche explicitement cyclostationnaire soit adoptée [6]. La figure 13a représente l'acquisition du signal vibratoire au niveau du clapet de refoulement côté fond sur un cycle machine en fonction de l'angle vilebrequin tel que préconisé ci-dessus. En correspondance avec la même base angulaire, la figure 13b montre l'évolution des pressions cylindres côté tige et côté fond $\left(p_{1}\right.$ et $\left.p_{2}\right)$ ainsi que des pressions en amont et en aval $\left(p_{u}\right.$ et $\left.p_{d}\right)$. Les évènements marqués $1,2,3$ et 4 se rapportent respectivement à l'ouverture et à la fermeture du clapet de refoulement, puis à l'ouverture et à la fermeture du clapet d'admission. L'indice $a$ désigne le côté fond et l'indice $b$ le côté tige. Bien que ces évènements soient difficilement identifiables sur le signal vibratoire, le spectre de Wigner-Ville en figure 13c permet assez clairement de les localiser dans le domaine angle-fréquence et de quantifier leur énergie vibratoire. Le suivi du spectre de Wigner-Ville constitue une stratégie de surveillance en tant que telle; des indicateurs scalaires simplifiés peuvent également en être déduits, tels que l'énergie dans un domaine angle-fréquence.

Exemple 6 : diagnostic des engrenages. Le diagnostic des engrenages repose sur de nombreuses méthodes qui sont implicitement liées aux notions de cyclostationnarité d'ordre 1 ou 2 (moyenne synchrone, analyse cepstrale, démodulation d'amplitude et de phase, etc.). Afin de prendre explicitement ces propriétés en compte, nous avons récemment proposé dans [2] de construire des indicateurs de cyclostationnarité d'ordre 1 et 2 dédiés à la surveillance des engrenages et définis comme suit :

$$
\left\{\begin{array}{l}
I C S_{1 x}=\sum_{\alpha \in \mathcal{A}, \alpha \neq 0}\left|\mathcal{P}_{\alpha}\{x(t)\}\right|^{2} / \mathcal{P}_{0}\left\{\left|x^{R}(t)\right|^{2}\right\} \\
I C S_{2 x}=\sum_{\alpha \in \mathcal{A}, \alpha \neq 0}\left|\mathcal{P}_{\alpha}\left\{\left|x^{R}(t)\right|^{2}\right\}\right| / \mathcal{P}_{0}\left\{\left|x^{R}(t)\right|^{2}\right\}
\end{array}\right.
$$

où $x^{R}(t)=x(t)-\mathcal{P}\{x(t)\}$. En posant $\mathcal{A}$ l'ensemble des harmoniques de rotation relativement à une roue dentée du système (et seulement cette roue), il est ainsi possible de mesurer les niveaux de cyclostationnarité d'ordre 1 et 2 développés par l'apparition d'un défaut sur cette roue. Cette stratégie autorise un diagnostic différencié de chacune des roues dans un système complexe. Elle est illustrée ci-dessous dans le cas d'un train d'engrenages à simple étage testé en fatigue accélérée au Centre Technique des Industries Mécaniques (CETIM Senlis) - cf. figure 14. La figure 15a représente l'évolution de l'indicateur de cyclostationnarité d'ordre $1-I C S_{1 x}-$ pour le signal de la roue menante filtré en deçà de $16 \mathrm{kHz}$ où les comportements périodiques prédominent, et la figure 15b l'évolution de l'indicateur de cyclostationnarité d'ordre $2-I C S_{2 x}$ - au-delà de $16 \mathrm{kHz}$ où le comportement est plutôt aléatoire. Dans les deux cas les indicateurs infléchissent une nette augmentation au cours de la $5^{\mathrm{e}}$ journée, ce qui correspond à l'apparition d'écaillage tel que constaté par les photographies de l'expert.

\section{Application à l'identification des systèmes mécaniques}

L'identification de systèmes mécaniques englobe plusieurs domaines d'application tels que l'analyse modale expérimentale, la caractérisation de matériaux, la validation de lois de comportement, le recalage de modèles numériques ou la modélisation phénoménologique de 


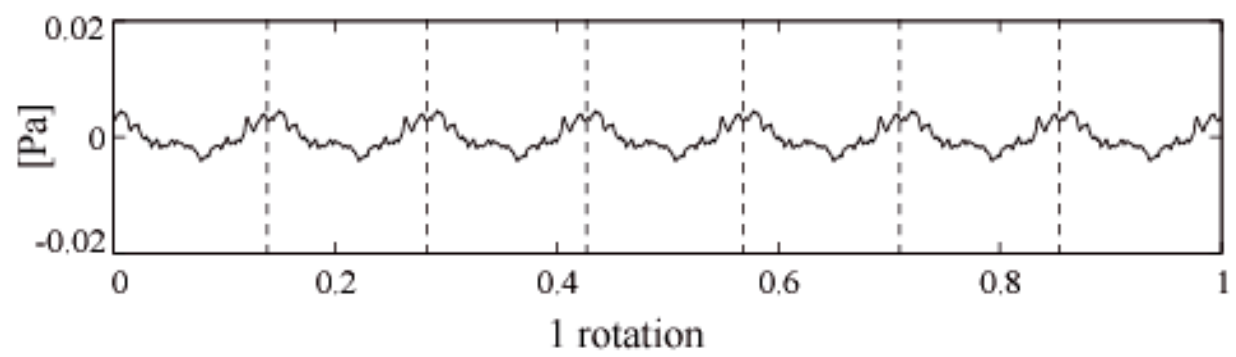

Fig. 11. Moyenne synchrone du signal de la figure 9 sans rééchantillonnage angulaire préalable.

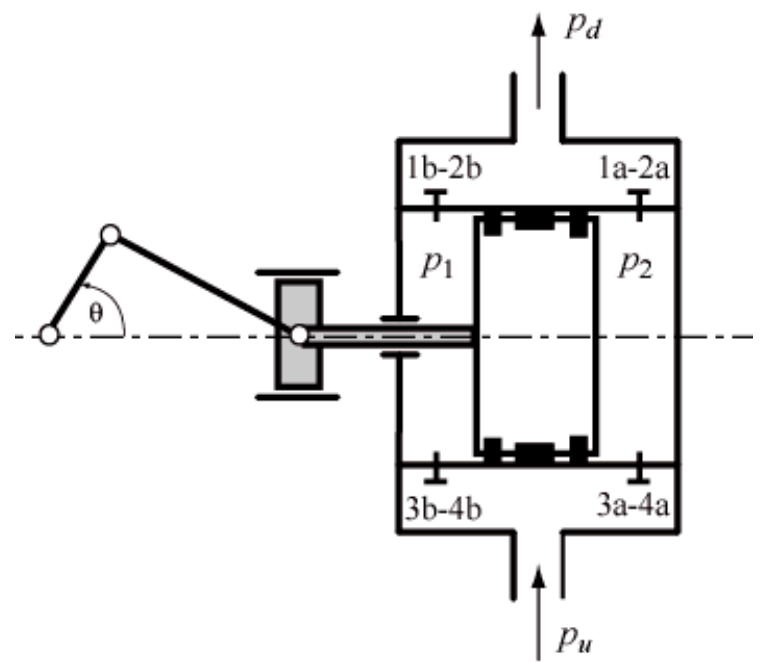

Fig. 12. Schéma d'un compresseur alternatif à double effet.

phénomènes complexes. Les systèmes mécaniques se caractérisent par certaines difficultés souvent absentes des autres disciplines : modèles de grande complexité, sources d'excitation internes difficiles à mesurer de façon nonintrusive et présence de nombreuses sources perturbatrices pour les mesures en fonctionnement. Dans ce contexte, la cyclostationnarité ouvre quelques perspectives intéressantes, telles que :

- une excellente immunité contre le bruit de mesure stationnaire en entrée et en sortie;

- la possibilité de focaliser l'identification par rapport à une source d'excitation cyclostationnaire spécifique indépendamment des autres sources de perturbation;

- l'utilisation de plusieurs fréquences cycliques afin d'augmenter le nombre d'observations par rapport au nombre d'inconnues du problème et donc de mieux conditionner le système (en particulier pour l'identification « aveugle »).

Exemple 7 : analyse modale expérimentale. L'analyse modale expérimentale consiste à identifier les paramètres modaux d'une structure (fréquences et modes propres, taux d'amortissement) à partir de ses réponses vibratoires à des excitations externes. Cela commence en général par la mesure, qui se doit d'être aussi précise que possible, des fonctions de réponse fréquentielles entre tous a)
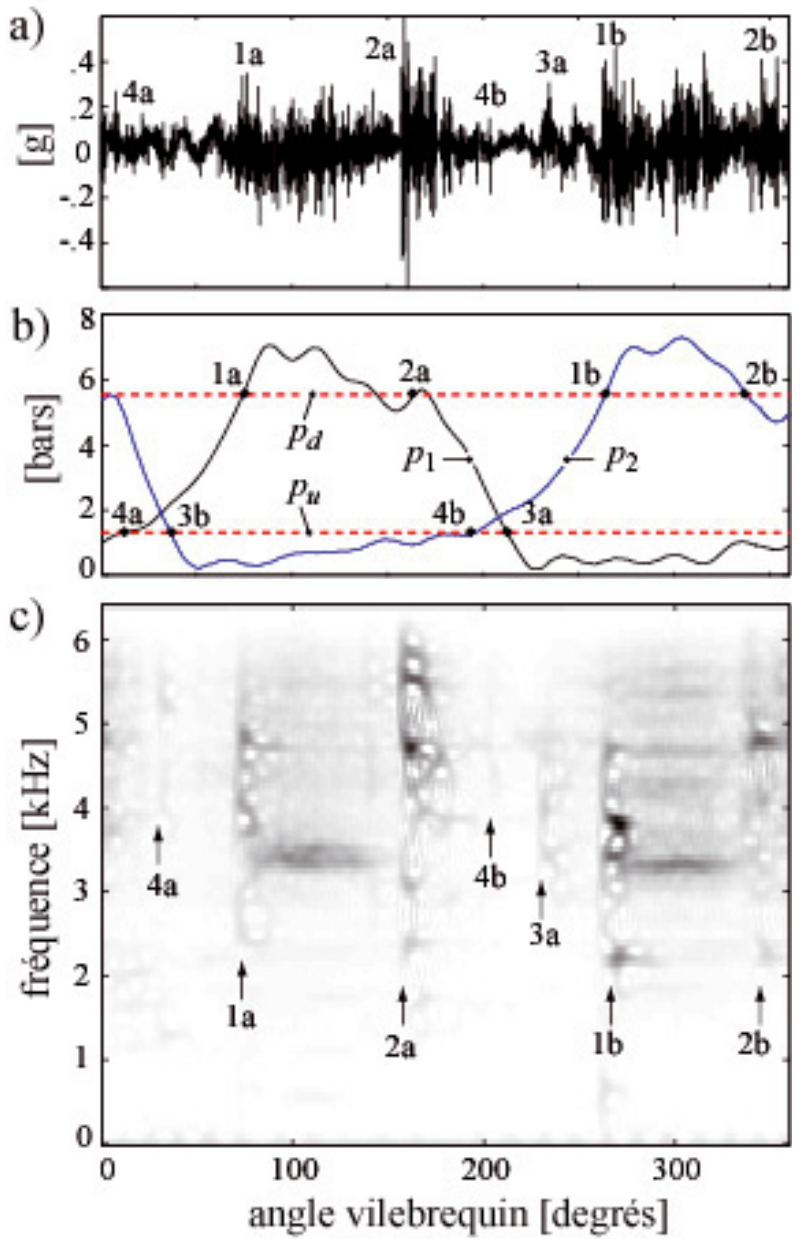

Fig. 13. (a) Signal vibratoire mesuré sur un compresseur et courbes de pression correspondantes. (b) Spectre de WignerVille du signal vibratoire $\left(\Delta \theta=10^{\circ} ; \Delta f=300 \mathrm{~Hz}\right)$.

les couples de points \{réponse-excitation\}. Étant donné une excitation $u(t)$ et une réponse $\nu(t)$ telles qu'illustrées à la figure $16 \mathrm{a}$, la problématique consiste à mesurer la réponse fréquentielle définie par

$$
H(f)=\lim _{\Delta f \rightarrow 0} \frac{v_{\Delta f}(t ; f)}{u_{\Delta f}(t ; f)}
$$

à partir des mesures bruitées $x(t)$ et $y(t)$. En raison des bruits d'entrée et de sortie $n_{i}(t)$ et $n_{o}(t)$, on montre que les estimateurs classiques $\left(H_{1}, H_{2}, H_{\nu}\right)$ sont biaisés 


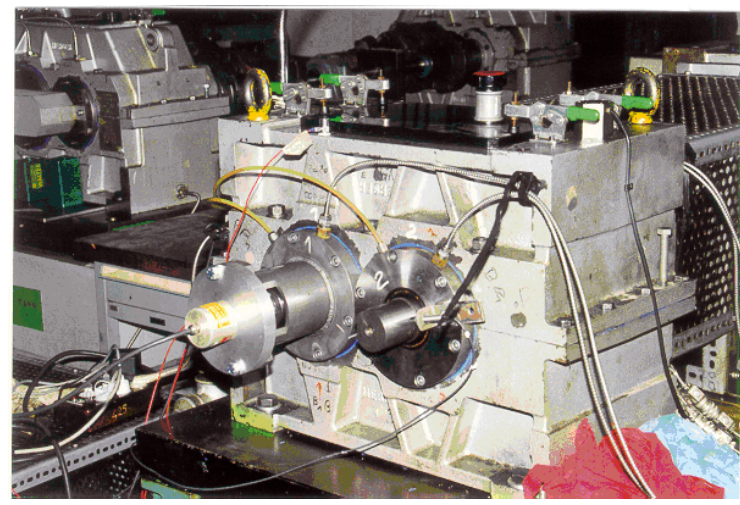

Fig. 14. Photographie du banc d'essais à un train d'engrenages (CETIM Senlis).

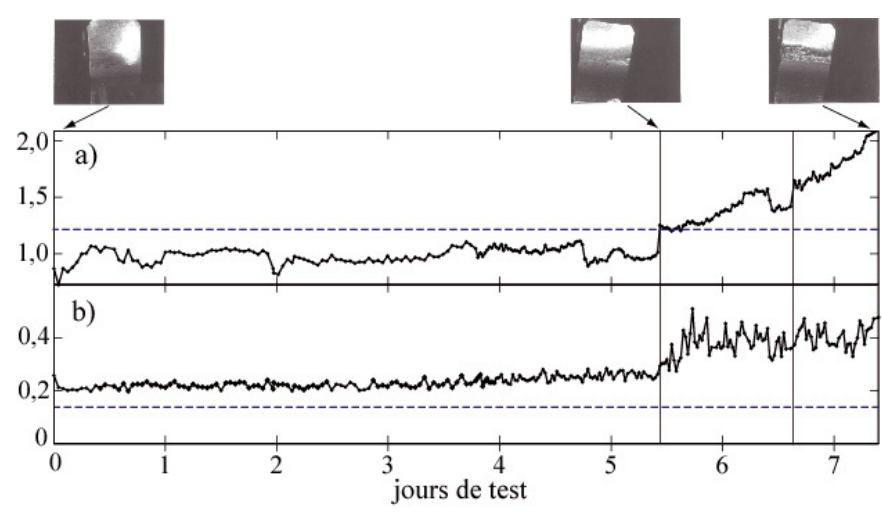

Fig. 15. (a) Évolution de l'indicateur de cyclostationnarité d'ordre 1 en deçà de $16 \mathrm{kHz}$ et (b) de l'indicateur de cyclostationnarité d'ordre 2 au-delà de $16 \mathrm{kHz}$ en fonction des jours de test. Les traits pointillés horizontaux représentent les seuils de significativité à $1 \%$.

et donc inappropriés dans ce contexte [7]. En exploitant le fait que les bruits de mesure $n_{i}(t)$ et $n_{o}(t)$ sont généralement stationnaires, il est cependant possible d'annihiler leurs effets à partir d'une excitation cyclostationnaire, par exemple de type «burst-random» telle qu'illustrée en figure 16b. On montre alors (voir Réf. [8]) qu'un estimateur non-biaisé de la réponse fréquentielle s'obtient en faisant

$$
H_{\alpha}(f)=\frac{C S_{y x}^{\alpha}(f-\alpha / 2)}{C S_{x}^{\alpha}(f-\alpha / 2)}
$$

où

$$
C S_{y x}^{\alpha}\left(f-\frac{\alpha}{2}\right)=\lim _{\Delta f \rightarrow 0} \frac{1}{\Delta f} \mathcal{P}_{\alpha}\left\{y_{\Delta f}(t ; f) \overline{x_{\Delta f}(t ; f-\alpha)}\right\}
$$

Ce résultat se justifie parce que $C S_{y x}^{\alpha}=C S_{\left(\nu+n_{0}\right)\left(u+n_{i}\right)}^{\alpha}=$ $C S_{\nu u}^{\alpha}$ où l'on a utilisé le fait que (1) $n_{0}$ et $n_{i}$ sont mutuellement décorrélés, de même que (2) $\nu$ et $n_{0}$ et (3) $u$ et $n_{i}$, par définition du bruit, et enfin (4) $C S_{n_{0}}^{\alpha}=C S_{n_{i}}^{\alpha}=0$ puisque le bruit est stationnaire. Pour les mêmes raisons, $C S_{x}^{\alpha}=C S_{u}^{\alpha}$, d'où finalement $H_{\alpha}=C S_{\nu u}^{\alpha} / C S_{u}^{\alpha}=H$. Il faut noter que la présence de bruit en sortie et en entrée (a)

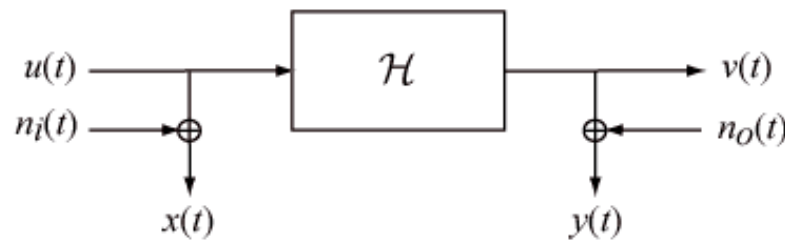

(b)

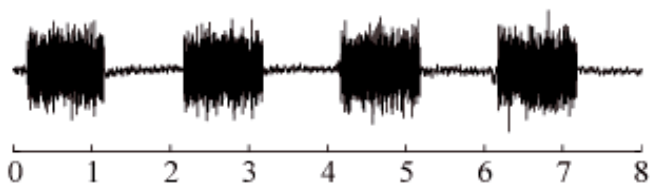

(c)

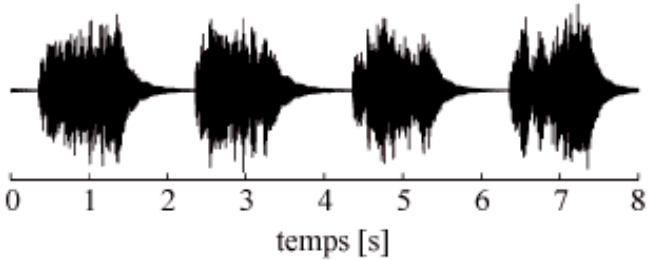

Fig. 16. (a) Représentation systémique de la problématique d'identification. (b) Excitation cyclostationnaire d'une poutre métallique avec (c) sa réponse vibratoire.

n'affecte pas cet estimateur, contrairement aux estimateurs classiques qui opèrent en contexte stationnaire et qui sont alors biaisés. Les résultats de cet estimateur sont comparés à ceux des approches classiques à la figure 17 pour la mesure de la réponse fréquentielle d'une poutre métallique (acier, $1000 \times 50 \times 8 \mathrm{~mm}$ ). Il apparaît clairement que la solution cyclostationnaire est sans biais, en particulier dans les zones critiques de résonance et d'antirésonance. Une extension de cette approche à l'analyse modale opérationnelle est présentée dans [9] lorsque, pour des contraintes pratiques ou pour des raisons de coût, l'excitation n'est pas maîtrisée. Dans ce cas, si une des sources d'excitation est cyclostationnaire (par exemple l'excitation appliquée par un moteur sur un wagon de train), les réponses vibratoires qu'elle génère peuvent être isolées sur la base de leur cyclostationnarité : il vient alors $C S_{y}^{\alpha}(f) \approx$ $H(f) H(f-\alpha) C S_{x}^{\alpha}$. Sous l'hypothèse supplémentaire que le temps de relaxation de la réponse modale est long devant le temps de corrélation de la source d'excitation, $C S_{x}^{\alpha}$ est à peu près constant dans le voisinage des modes de sorte que $C S_{y}^{\alpha}(f) \propto H(f) H(f-\alpha)$, formule qui donne accès à la fois au module et à la phase de la fonction de réponse en fréquence $H(f)$ à l'indétermination près du facteur d'amplitude (complexe) $C S_{x}^{\alpha}$.

\section{Application à la séparation de sources mécaniques}

La séparation de sources est un problème inverse qui consiste à reconstruire les effets individuels de chacune des sources d'excitation qui participent à la réponse globale d'un système. La problématique est d'intérêt dans toutes les applications où les sources ne peuvent pas être isolées expérimentalement. C'est en particulier le 

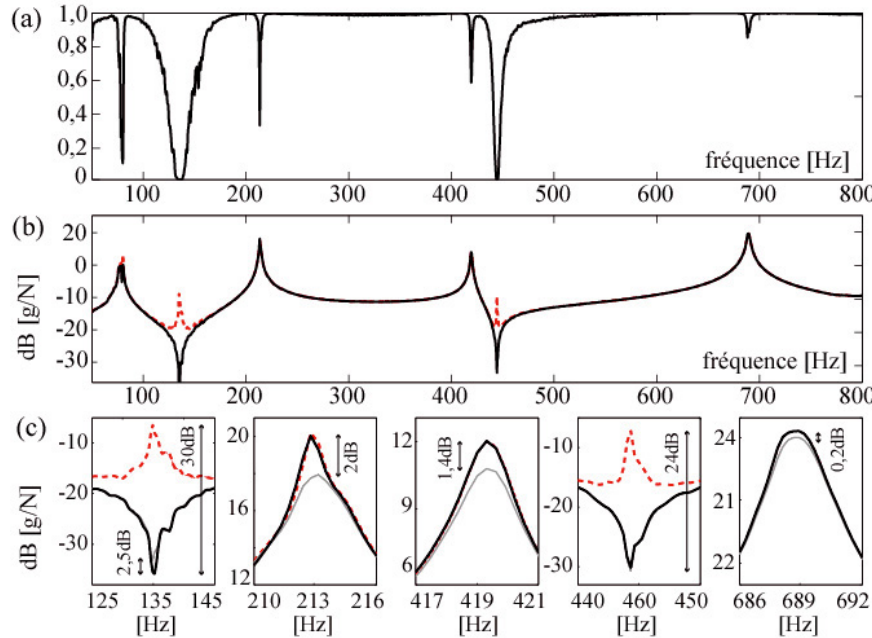

Fig. 17. (a) Fonction de cohérence entre l'excitation $x(t)$ et la réponse vibratoire $y(t)$. (b), (c) Comparaison entre l'estimateur $H_{1}$ (traits pointillés en gris), l'estimateur $H_{2}$ (traits pointillés en rouge) et l'estimateur cyclostationnaire $H_{\alpha}$ (trait continu en noir) de la réponse fréquentielle. $(\Delta f=39 \mathrm{~Hz})$.

cas des machines sur site dont le fonctionnement implique la présence de nombreuses sources concurrentes qu'il n'est pas possible de mesurer individuellement de manière non-intrusive. Ici encore, la propriété de cyclostationnarité peut être avantageusement mise à profit dans le cas de sources cyclostationnaires pour apporter l'information supplémentaire nécessaire à la résolution du problème de séparation. Nous en donnons ici un aperçu succinct au travers de deux cas d'études.

Exemple 8 : séparation supervisée de sources acoustiques. La décomposition du bruit global rayonné par les moteurs automobiles en leurs contributions élémentaires dues au bruit de combustion, au bruit d'injecteur (moteurs Diesel) et au bruit mécanique (basculement du piston) est une problématique récurrente qui illustre très bien les objectifs de la séparation de sources. La figure 18a montre le signal acoustique rayonné au droit de la face avant d'un moteur Diesel à 4 cylindres, tournant à 1200 tr.min ${ }^{-1}$, sur un intervalle angulaire de $100^{\circ}$ centré autour du point mort haut du premier cylindre. L'objectif est de décomposer ce signal $y(t)$ en une somme de contributions $x_{i}(t), i=1,2,3$ provenant respectivement du bruit de combustion dans le cylindre, du bruit d'injecteur et du bruit mécanique résiduel tel que

$$
y(t)=\sum_{i=1}^{3} x_{i}(t)
$$

Étant donné des mesures de référence $r_{i}(t), i=1,2$ sur le processus de combustion (capteur de pression cylindre) et sur le processus d'injection (capteur piézoélectrique sur la pompe d'injection), nous avons montré dans [10] qu'il est possible d'extraire chacune des contributions à l'aide du filtrage « cyclique »

$$
x_{i, \Delta f}(t ; f)=\sum_{k=0}^{K} H_{i}^{\alpha_{k}}(f) r_{i, \Delta f}\left(t ; f-\alpha_{k}\right) \mathrm{e}^{j 2 \pi \alpha_{k} t}
$$
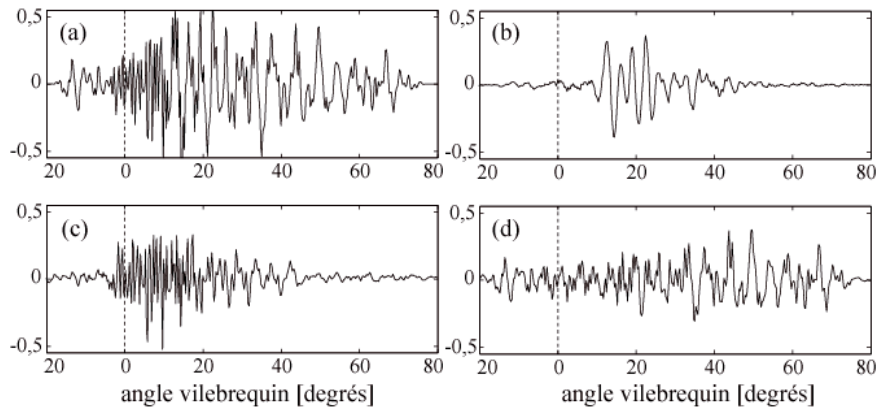

Fig. 18. (a) Bruit global rayonné par le moteur et sa décomposition en (b) bruit de combustion, (c) bruit d'injecteur et (d) bruit mécanique résiduel. (a)

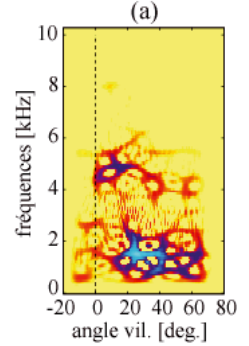

(b)

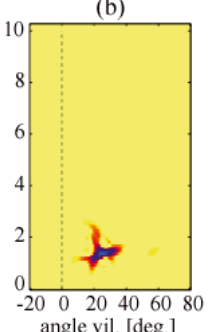

(c)

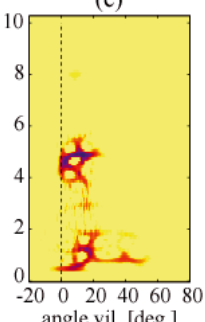

(d)

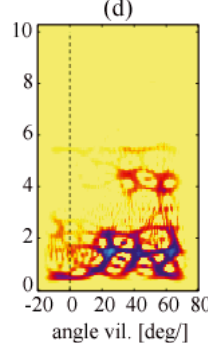

Fig. 19. Spectres de Wigner-Ville associés aux résultats de la figure $18 \mathrm{a}-\mathrm{d}$.

sur les fréquences cycliques $\alpha_{k}, k=0, \ldots, K,\left(\alpha_{0}=0\right)$ de la source $i$, où les coefficients fréquentiels $H_{i}^{\alpha_{k}}(f)$ sont obtenus par résolution du système d'équations

$$
\begin{aligned}
& C S_{y r_{i}}^{\alpha_{l}}\left(f-\frac{\alpha_{l}}{2}\right)=\sum_{k=0}^{K} H_{i}^{\alpha_{k}}(f) S C_{r_{i}}^{\alpha_{l}-\alpha_{k}}\left(f-\frac{\alpha_{k}+\alpha_{l}}{2}\right) \\
& l=0, \ldots, K
\end{aligned}
$$

Les résultats de la séparation sont présentés aux figures $18 \mathrm{~b}-\mathrm{d}$ pour les formes d'onde et à la figure 19 pour les spectres de Wigner-Ville correspondants. Ils sont parfaitement pertinents en termes de localisation angulaire et fréquentielle des phénomènes source.

Exemple 9 : séparation aveugle de sources vibratoires. Disposer de références sur les sources dont on cherche à extraire les contributions est un luxe inaccessible dans beaucoup de situations. Dans le cas où les sources ne partagent pas les mêmes fréquences cycliques, il est cependant possible d'extraire leurs contributions en aveugle suivant une démarche très similaire à la précédente [11-14]. Soit $x_{j \mid i}(t)$ la contribution de la source $i$ dans la mesure $y_{j}(t)$ au capteur $j, j=1, \ldots, m$. Si la source $i$ se caractérise par un ensemble de fréquences cycliques $\alpha_{k}, k=0, \ldots, K\left(\alpha_{0}=0\right)$ qui lui sont propres, alors on montre que

$$
x_{j \mid i, \Delta f}(t ; f)=\sum_{j=1}^{m} \sum_{k=1}^{K} K_{i j}^{\alpha_{k}}(f) y_{j, \Delta f}\left(t ; f-\alpha_{k}\right) \mathrm{e}^{j 2 \pi \alpha_{k} t}
$$




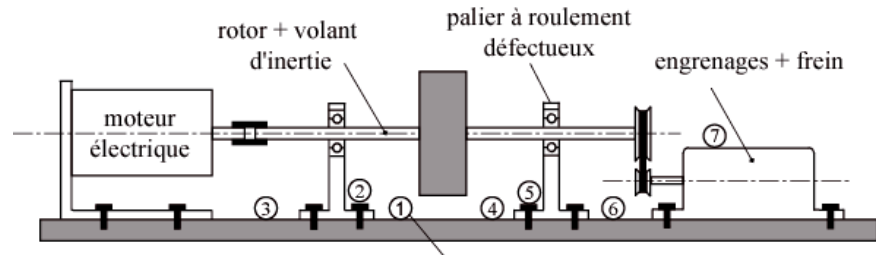

accéléromètre 1

Fig. 20. Banc d'essai de machine tournante avec défaut de roulement sur le palier droit. Les positions des capteurs accélérométriques sont indiquées par leurs numéros.

a)

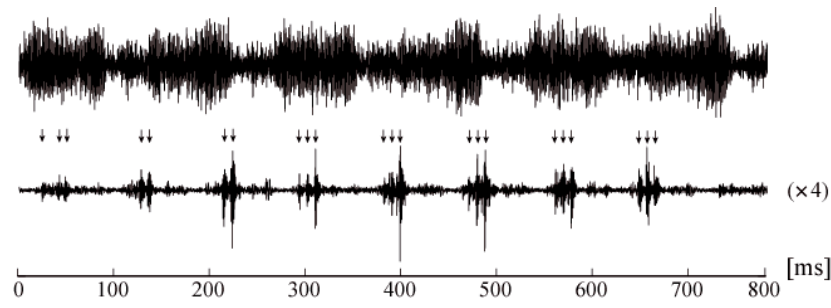

Fig. 21. (a) Signal vibratoire mesuré au capteur 1. (b) Contribution du défaut de roulement amplifiée d'un facteur 4 avec indication des instants où la bille défectueuse impacte les pistes interne et externe.

où l'on a pris soin de ne pas inclure la fréquence cyclique nulle $\alpha_{0}$ et où les coefficients fréquentiels $K_{i j}^{\alpha_{k}}(f)$ sont solutions du système d'équations

$$
\begin{aligned}
& C S_{y_{j} y_{q}}^{\alpha_{l}}\left(f-\frac{\alpha_{l}}{2}\right)=\sum_{k=1}^{K} H_{i j}^{\alpha_{k}}(f) S C_{y_{j} y_{q}}^{\alpha_{l}-\alpha_{k}}\left(f-\frac{\alpha_{k}+\alpha_{l}}{2}\right) \\
& l=1, \ldots, K, q=1, \ldots, m
\end{aligned}
$$

Cette stratégie est illustrée ici pour extraire la (très faible) contribution d'un défaut de roulement à billes dans le signal vibratoire global mesuré sur un banc d'essai de machine tournante - cf. figure 20. Sept signaux vibratoires $y_{j}(t), j=1, \ldots, 7$ ont été acquis en des endroits relativement éloignés du palier qui supporte le roulement défectueux. La figure 21a montre le signal $y_{1}(t)$ au capteur 1, qui se caractérise par une forte activité vibratoire essentiellement due à la rotation du moteur électrique, du rotor et au fonctionnement du réducteur à engrenages. La cohérence spectrale de ce signal (non représentée ici) permet d'identifier la fréquence de passage du défaut de bille à $116 \mathrm{~Hz}$ avec ses raies latérales espacées de $29,7 \mathrm{~Hz}$, la fréquence de rotation du rotor, ainsi que la fréquence de rotation de la cage du roulement à $11,3 \mathrm{~Hz}$ avec ses harmoniques. Le signal extrait en figure $21 \mathrm{~b}$ a été obtenu en prenant $\alpha_{2 k}=116 k, \alpha_{2 k+1}=-116 k, k=1, \ldots, 6$. À noter que le signal du défaut est si faible par rapport au niveau vibratoire global que nous avons dû l'amplifier d'un facteur 4. Des résultats similaires ont été obtenus sur les autres capteurs.

\section{Conclusion}

De nombreux signaux mécaniques, et tout spécialement les signaux vibratoires et acoustiques, se décrivent étonnamment bien dans le formalisme cyclostationnaire qui a été développé au cours des dernières décennies dans le domaine des télécommunications. L'objectif de cet article a été de dresser un bref panorama des possibilités que l'on peut attendre de ce nouveau formalisme. Les exemples que nous avons présentés touchent au diagnostic, à l'identification et à la séparation de sources, mais nous espérons qu'ils ouvriront également des perspectives dans d'autres domaines d'application.

Une des conditions nécessaires pour tirer pleinement parti du formalisme cyclostationnaire est que les mécanismes qui le génèrent (par exemple la rotation d'une machine) opèrent à vitesse de rotation rigoureusement constante. En présence de petites fluctuations de vitesse autour d'une vitesse nominale, cette condition peut être forcée par un rééchantillonnage du signal dans le domaine angulaire à partir d'une référence de phase. Le formalisme cyclostationnaire n'est théoriquement plus valable en présence de grandes variations de régime (montée ou descente de régime) et sa généralisation dans ce contexte reste à construire.

\section{Références}

[1] W.A. Gardner, Introduction to Random Processes, McGraw-Hill, 2nd Ed., 1990

[2] A. Raad, J. Antoni, M. Sidahmed, Indicators of cyclostationarity: Theory and application to gear fault monitoring, Mechanical Systems and Signal Processing 22 (2008) 574-587

[3] J. Antoni, R.B. Randall, Differential Diagnosis of Gear and Bearing Faults, ASME J. Vib. Acoust. 124 (2002) $165-171$

[4] J. Antoni, F. Bonnardot, A. Raad, M. El Badaoui, Cyclostationary Modelling of Rotating Machine Vibration Signals, Mechanical Systems and Signal Processing 18 (2004) 1285-1314

[5] J. Antoni, R.B. Randall, A Stochastic Model for Simulation and Diagnostics of Rolling Element Bearings with Localized Faults, ASME J. Vib. Acoust. 125 (2003) 282-289

[6] R. Zouari et al., Cyclostationary Modelling of Reciprocating Compressors and Application to Valve Fault Detection, Int. J. Acoust. Vib. 12 (2007) 116-124

[7] D. Ewins, Modal Testing - Theory, practice and Application, 2nd Ed. Research Studies Press, 2000

[8] J. Antoni, P. Wagstaff, J.-C. Henrio, $H_{\alpha}-$ A Consistent Estimator for Frequency Response Functions with Input and Output Noise, IEEE Instrum. Meas. 53 (2004) 457465 
[9] D. Hanson, R.B. Randall, J. Antoni, D.J. Thompson, T.P. Waters, R.A.J. Ford, Cyclostationarity and the cepstrum for operational modal analysis of mimo systems - Part I: Modal parameter identification, Mechanical Systems and Signal Processing 21 (2007) 2441-2458

[10] J. Antoni, M. Sidahmed, F. Gautier, S. Wang, Séparation et hiérarchisation des sources de bruits dans les moteurs par filtrage cyclique, $\mathrm{XV}^{\mathrm{e}}$ colloque, Vibrations Chocs \& Bruit, 14-16 juin 2006, École Centrale de Lyon

[11] F. Bonnardot, J. Antoni, R.B. Randall, M. El Badaoui, Enhancement of Second Order Cyclostationary Signals, Int. Conf. on Acoustics, Speech, and Signal Processing, Montreal, Canada, 2004
[12] F. Bonnardot, R.B. Randall, F. Guillet, Extraction of second-order cyclostationary sources - Application to vibration analysis, Mechanical Systems and Signal Processing 19 (2005) 1230-1244

[13] R. Boustany, J. Antoni, A Subspace Method for the Blind Extraction of a Cyclostationary Source: Application to Rolling Element Bearing Diagnostics, Mechanical Systems and Signal Processing 19 (2005) 1245-1259

[14] R. Boustany, J. Antoni, Blind extraction of a cyclostationary signal using reduced-rank cyclic regression A unifying approach, Mechanical Systems and Signal Processing 22 (2008) 520-541 